\title{
Preservation or reincarnation? At the crossroads of ethical values
}

\author{
Dr. Bernhard Serexhe \\ Karlsruhe \\ https://serexhe.net/
}

\section{Keynote}

For both the artist and the viewer, or user, digital technology has expanded creative possibilities and horizons, only to narrow them again at the same time. We have blindly embraced digitization, enjoying its promising benefits to which we have willingly adapted. The price we all pay for the tantalizing possibilities of virtual and augmented realities is the loss of immediate sensory contact with reality. It almost seems as if digitization has discredited the analog world as essentially inadequate. However, in an endless evolutionary process, humanity has developed sensory capabilities to interpret the analog world, whereas today we have become essentially dependent on machine processing and interpretation to understand and advance our world through digital data. In our consumer-driven economy of obsolescence, we have been overrun by this development without being able to protect ourselves.

This is not the place to deal in extenso with the philosophical issues of this societal and technological paradigm. Nevertheless, as we cannot escape the fact, that our life and work depends on the very implications of these questions. For it has been proving for decades that the innovations imposed by a thriving IT and communications industry run so fast ahead of a fundamental reflection on what we actually want, that in every situation we find ourselves confronted with the serious consequences that we then struggle to repair. On a much larger scale, this is visible in climate change, in the work environment, and in the feeling of senselessness of a society "amusing itself to death". And it is becoming abundantly clear, for example, in the comprehensive digital surveillance of individuals and society by state institutions and large, globally operating private companies. 
It is equally reflected in the changes in the art world, and especially in our working conditions as curators and conservators. In the light of this change, the core of theories and practices in the arts must also be reevaluated and traditional standards called into question. ${ }^{1}$ This questioning has been necessitated

by the advent of digital tools and transmission media that have also fundamentally changed the conditions of production and distribution in art. ${ }^{2}$ The conventional terms that art historians, curators and conservators have always used to describe art have long since become problematic when it comes to precisely defining and delimiting their meaning. The categories and genres used in previous forms of art historical analysis no longer apply; they are unable to reliably capture the changes that have occurred. ${ }^{3}$

In recent decades, the traditionally proven tasks of museums and collections collecting, preserving, researching and mediating - have been confronted with an art whose performative, process-oriented, even ephemeral and at the same time global character virtually resists safe preservation or transmission as cultural heritage. ${ }^{4}$ This

\footnotetext{
${ }^{1}$ Thus, the self-image of our societies has been shaped for millennia by stable systems of passing on customs and traditions that are geared to longevity. In his studies of ancient Egyptian culture, the Egyptologist and scholar of religion and culture Jan Assmann has convincingly demonstrated that "the tradition within us, [...] solidified over generations, over centuries, even millennia of repeated texts, images, and rites, [...] shapes our consciousness of time and history, our view of ourselves and the world." Jan Assmann, "Cultural Memory," in Thomas Mann and Egypt: Myth and Monotheism in the Joseph Novels (Munich: C. H. Beck, 2006), 70. Crucial to this conclusion is the argument that "cultural memory" (a term coined by Assmann) in all previous civilizations has been geared toward longevity and reliability, especially in response to the constant threat of external influences, wars, targeted destruction, natural disasters, and natural processes of decay.

${ }^{2}$ At all times and in all societies, cultural developments are closely intertwined with the economic, political, and social conditions that both determine them and result from them. Therefore, we must strive for a consideration that goes beyond the usual horizon of political, conservation, or stylistic evaluations of a collection. Only against this broader horizon, which asks more generally about the interdependencies of culture and economy, can phenomena be understood that, on closer examination, can all too easily be attributed to the imperfection of individual technologies or the failure of individual institutions.

${ }^{3}$ As early as 1983, Hans Belting posited that "that contemporary art indeed manifests an awareness of a history of art but no longer carries it forward; and that the academic discipline of art history no longer disposes of a compelling model of historical treatment." Hans Belting, The End of Art History, trans. Christopher S. Wood (Chicago, 1987), 3; first published as Das Ende der Kunstgeschichte (Munich, 1983). See also Belting, Das Ende der Kunstgeschichte - Eine Revision nach zehn Jahren (Munich, 1995); not available in English translation.

${ }^{4}$ In his 1928 essay "La conquete de l'ubiquité," Paul Valéry foretold the coming transformation of art through new technologies: "We must be prepared for great innovations to reshape the whole technique of the arts,
} 
development not only affects works of media art, but also began at the latest with the performances of the Dada movement after World War I against the hypocritical bourgeois cultural ideals of quality, truth, and beauty. And it has continued in all the avant-garde art movements of the 20th century, which opposed the trivializing comodification of art in bourgeois society.

While these artists systematically appropriated ephemeral materials ${ }^{5}$, and employed new techniques and forms of presentation, rapid technological change led to a technical obsolescence that can be seen as a systemic threat to art as long as we remain faithful - in the fine old tradition - to the categories of enduring value and longevity. Long before the rampant digitalization of all human expressions of life, including art, artists systematically used materials and organisms (biological and chemical substances), electronic tools (cameras, microphones, interfaces), technical storage media (records, magnetic tapes, laser discs) as well as presentation and transmission techniques (performance, radio, video, television) whose durability is fundamentally in question due to unstoppable biological, chemical, physical and/or technical processes as well as the potential lack of availability of the necessary reproduction devices on the market. Private and public collections, often unprepared for this to date, have had to face the recognition that many of their works are acutely threatened with decay.

Another type of acceleration, which in this case can be considered absolute, leads to the decay of works of art due to the irreversible system change from analogue to digital technologies. This development applies regardless of whether these works have been digitised to copy, simulate and store their original analogue data (graphic documents, photographs, films, videos), whether they have been produced through the direct use

and thus to affect artistic invention itself, and perhaps even to bring about an astonishing change in our concept of art." Paul Valéry, "The Conquest of Ubiquity," trans. Ralph Manheim (New York: Pantheon Books, Bollingen Series, 1964), 225; first published as "La conquête de l'ubiquité," 1928.

${ }^{5}$ Chocolate, honey, fat, blood - the list of degradable materials is long. In 2002, Teresa Margolles exhibited her work Vaporization (2002) at PS1 in New York, in which the exhibition space is filled with steam generated from water previously used to wash corpses in a morgue in Mexico City. 
of digital apparatus (digital audio/video recording devices) or whether they are entirely the result of the programming of their source code. In the preservation of such works and cultural objects in general, shorter storage times and massively increased storage capacities have led archives and collections to take ever greater risks and sometimes even to abandon preservation in analogue form. The problem is that the storage and retrieval of digitized content is subject to new technical systems and constant software changes in rapid succession. The central promise of long-term digital data security has by no means been fulfilled by the computer and software industry.

From this we can conclude that it is not the digital works themselves that age faster than their analogue counterparts, because their very substance, their idea, is laid down in the digital code, which cannot age. Rather, we can observe that the devices and their operating systems, the interfaces, sensors and other specific applications, the authoring systems, plug-ins, players and browsers required to collect, preserve and present these works, the programming skills needed to adapt them, and often enough the financial resources to reactivate and present them again - are only available to a limited extent after just a few years. Public and private collections have recognised too late what this problem means for media art anchored in the digital code: the faster technical development advances, the shorter the half-life of the artworks. Based on the experience of the last three decades, we must today assume that digital hardware and software have a shelf life of less than ten years. The resulting functional obsolescence of digital artworks leads the previously valid collection criteria of durability, authenticity and lasting value ad absurdum and requires a general rethinking. Collections and museums will by no means be able to keep pace with technological developments if they maintain their previous structures and funding.

It is largely undisputed that the substance of art lies in the idea. But this underlying idea can only be expressed, conveyed, and comprehended by its visitors or users through a personal profound sensory experience. This definitely requires material media, be they cave walls, stone sculptures, paintings on canvas, photographs on paper, or even the devices on which the storage and reproduction of digital art depends - namely computers, hard drives, interfaces, sensors, monitors, projectors and all other apparatuses that function in the digital process but are anything but immaterial. 
Over the millennia, the awareness has prevailed that the information stored in analogue media can be retrieved as long as these media exist in their material form. The extremely short history of digital media has proven exactly the opposite with each cycle of "improvements" within just three decades. ${ }^{6}$

But against all critical insights, we continue to live in the jubilant phase of digitization. As if it were the most unavoidable of our duties, masses of historical documents and analogue art works of all kinds are being forcibly digitized all over the world to save them, as they say, from loss and perishing. However, almost no one is interested in the fact that this does not save the works themselves in their original form, but that the preserved digitized data is no more than a mediocre reduction, just an extremely vulnerable substitute for the original objects. Thanks to the powerful digital lobby, the one-way street from analogue to digital is basically presented as the golden road to solving conservation issues. Not even ICOM seriously questions the supposedly compelling need for these measures when drafting the new museum definition. But we as curators and conservators have to live with the risks and side effects and often do not have the knowledge and resources to do so.

\section{Prevailing Conditions of Preservation}

As the former director of the Media Museum and its large collection at the ZKM I Center for Art and Media Karlsruhe, Germany, and since 2016 as an international consultant to museums, exhibition and conservation projects, I am somewhat familiar with the prevailing conditions of exhibiting and preserving electronic and digital art. Over the past thirty years, the following picture has emerged for me, which I would like to briefly outline here without claiming to be complete:

\footnotetext{
${ }^{6}$ We should remember that just three decades ago, for example, so-called floppy disks were used to store and transfer digital data. In the repositories of many collections today, early digital works "slumber" on floppy disks and other digital storage media for which there are no longer any devices that can "revive" them. After just five years, data stored on CDs and DVDs can begin to fade or even become completely unreadable. Hard drives can fail at any time for a variety of reasons. To make matters worse, the outdated software needed to activate these works is also often no longer available or cannot be installed on today's computers.
} 
- Under pressure from public and private patrons and sponsors, many museums and collections have accepted to justify their funding by the means of accelerated marketing and advertising, constant up-to-date presence in social media and, above all, a steadily increasing number of short-term events and exhibitions. While higher visitor numbers have become the ultimate proof of eligibility for funding, there are fewer and fewer funds available for elaborate preservation measures that are barely visible from the outside and for building competent teams that are proficient in preserving the latest media.

- Museum directors and donors are still not sufficiently sensitized to the issues and costs of preserving digitized or digital born artworks. In the art market, media art still has a low status because gallery owners and potential collectors often fear the maintenance and preservation costs. It seems unattractive to pay similar prices for invisible data, whose visualization into a work of art requires a great deal of effort, as for paintings, whose presentation requires only a good room climate and the nail on which they hang.

- Finally, a funding policy demanded by the digital lobby has led to a preference for the development of digital processes, in the course of which it has become easier to obtain funding for the digitization of objects, works and documents than for the proper preservation of these objects themselves.

- In addition, many collections have not yet established an efficient archival system for digital art in which all movements, maintenance and preservation measures, technical interventions, and artist intentions for individual works can be recorded without gaps. The frequent absence of such archival structures has led to a serious lack of intermediate information that is essential for proper presentation and preservation.

- When acquiring and taking over those digital artworks in recent decades, which we find in existing collections today, not enough attention has often been paid to careful documentation due to a lack of experience. As late as the 1990s, it was naively assumed that immaterial digital data and its highly praised storage 
media would last forever. Some awareness of the great vulnerability of this data only emerged in the mid-2000s, when the rapid introduction of smartphone technology and the massive spread of apps led to a further expansion of artistic possibilities.

- In the process, one has all too often forgotten to document the work-defining instructions given by the artist. In other cases, a clear application of these instructions has proven to be too complicated or even impossible. It has become common practice to ask for the artist's opinion during exhibitions or restoration work, if the artist is willing and able to do so. However, we are often enough confronted with situations in which we have to protect the acquired work against the spontaneously changing intentions of the artist.

- Conservators and museum technicians are often overwhelmed with the complex daily maintenance of exhibited electronic or digital works and the continuous repair and preservation of those works that are not currently on display. The knowledge required for this is still extremely rarely part of their training. In addition, for many objects they would have to be supported by informaticians who are definitely prepared for dealing with digital art and whose recruitment is unfortunately not encouraged by an often restrictive personnel management.

- It is therefore fully understandable that conservators, who were already busy with the daily care and preservation of analogue artworks, were completely overburdened with the new additional task of preserving digital and video art, for which they were not trained. Thus, in the past, tasks that definitely belong to the museum's domain, for example the digitization and restoration of genuinely analogue video art, were often left to the nimble emerging branch of external service providers who saw it as merely a technical task to be solved. The consequence of this is very often that works already incorporated into the collections have had their functioning and aestetics compromised by incompetent, cheap and poor treatment. 
- Last but not least, many collections lack the appropriate technical facilities and laboratories to maintain, preserve, and if necessary restore digital or digitized art. These facilities could not even be set up without qualified personnel if the funds were available. From this point of view, the hesitation of many museum directors and collectors to acquire and exhibit digital art becomes understandable; the associated risks and costs seem too high in times of shrinking budgets.

These selected observations of our working conditions may sound like a complaint. But they give a concerned overview of those institutional, technical and personnel conditions and restrictions under which our work often takes place. As a researcher, one can spend years looking at things from a variety of angles and putting them into perspective. As a responsible technician and conservator, and as a curator too, one must be able to act competently every day, here and now, and have the necessary resources to do so. I would be happy to hear later from the floor that at some facilities conditions are sometimes much better.

\section{At the crossroads of ethical values}

It has long become obvious, that the previously upheld ethical values underlying the preservation of art works and cultural heritage in general must be fundamentally renegotiated. ${ }^{7}$ Although historically there has never been a strict codification of such values, curators and conservators have always relied on the widespread agreement that works of art are original, unique, authentic, have high value, and are endowed with a distinctive work character. In an idealized view, it was also firmly postulated that works of art, as long as they preserved these qualities, bore witness to that kind of historicity in which the cultural history of mankind could be found. For this noble ethical reason and, no less of course, for the further development of the financial and market value, the conservation process should aim, with the utmost respect, to

\footnotetext{
${ }^{7}$ Important research in this field is for instance done by Lino Garcia Morales in his Theoria de la Conservation Evolutiva, published 2019.
} 
preserve these qualities as long as possible in their unaltered original form, their native material and with the highest sensitivity to the impression and intention conveyed by the hand of the artist. This has been a dream for a long time.

But already with the advent of analogue video art in the 1970s and even more so with the advent of digital video and digital born art since the 1990s, the inconsistencies with traditional conservation ethics were recognized, and various research programs have addressed the question of changing ethical values in conservation. ${ }^{8}$ The following quote is found in the 2010 article Ethics and practices of media art conservation, a work-inprogress written by researcher Gaby Wijers, one of the most valuable publications on the subject:

"In fine art conservation, the main ethical issue is that all conservation activities should be faithful to the integrity of the original art object, respecting both the authentic, original appearance of the work and the artist's original intention. Most ethical codes specify different kinds of integrity: physical, aesthetic and historical. The first refers to the material components, the second describes the ability of an object to create an aesthetical sensation for the viewer, and the last describes the history that has imprinted on the object. These concepts of integrity (appearance and intention) are the greatest and most urgent issues confronting the field of media art conservation." ${ }^{9}$

\footnotetext{
${ }^{8}$ Electronic Arts Intermix, 1971; symposion Modern art who cares, 1997; Variable Media Initiative, 1999; DOCAM, Documentation and Conservation of the Media Art Heritage, 2005; Matters in Media Art, 2005; Inside Installations: Preservation and Presentation of Installation Art, 2004-2007; Digital Art Conservation. Preservation of Digital Art: Theory and Practice, 2010-2014, including the two symposia: - The Digital Oblivion: Substance and Ehtics in the conservation of Computer-based Art, ZKM | Karlsruhe, 2010, and - Digital Art Conservation. Practical Approaches: Artists, Programmers, Theorists, Strasbourg, 2012; symposion: Re: New Media Art. Technologybased art conservation, Amsterdam 2019

${ }^{9}$ Gaby Wijers (Netherlands Media Art Institute), Ethics and practices of media art conservation, a work-in-progress (version0.5), Publication date: August 2010, quoted on https://www.scart.be/?q=en/content/ethics-and-practices-media-art-conservation-workprogress-version05
} 
At the time of this quoted publication, it was clear to its author that the discussion of ethical values in conservation is a permanent "work-in-progress". After the extensive discussions of the topic so far, let us consider whether and how, under today's conditions, adherence to the value of "faithfulness to the integrity of the original art object" is possible and still meaningful.

\section{The value of physical integrity}

With the rapid progress of technical development, we are experiencing that the technical devices used in works of art, such as computers, monitors, projectors, cameras, hard disks, interfaces, etc., which function in works of art in absolute dependence on one another, can no longer be replaced in their original form after five to ten years at the latest. Under the pressure of an economy geared to constant renewal, they are replaced at short intervals by a new generation that only approximates their former functions. The same applies to the further development and change of operating systems. The consequences for the preservation of media artworks have been described so frequently and precisely so far that they do not need to be explained further here.

In terms of the ethical value of maintaining the physical integrity of an artwork, the constant obsolescence of equipment is highly detrimental. To be clear, even if we had the means to repair and extend the life of equipment by keeping it in storage or buying it on the second-hand market, under these conditions physical integrity can no longer be preserved even in the medium term. It seems ironic in this context to recall that for centuries our best collections have managed to preserve important and less works of art for the present day.

A solution of this well-known problem could be that we increasingly concentrate on the transmission of the underlying idea of the work and in return accept a partial or even far-reaching modification of its physical integrity. This is admittedly connected with the highest risks also for the transmission of the esthetic and historical integrity. But if we are confronted with the total absence of specific industrially produced hardware, as has 
long been the case with analogue video or laser disc players and in particular with CRT monitors, then even increasingly elaborate repairs will only be able to prolong the life of such products to a limited extent. As curators and conservators, however, we are trained to think in terms of decades, if not centuries, and it is precisely this large time span that is now seriously in question.

Example: In 2018, I was commissioned to do a preparatory study for the restoration of Nam June Paik's extensive work The More the Better of 1988, at the Museum for Modern and Contemporary Art, Gwacheon, Gyeonggi Province, South Korea. This work can be described as an impressing multichannel video architecture, consisting of 1,003 CRT monitors of different sizes, arranged like a cone 18 meters high, weighing 16 tons and equipped with wiring that largely dates from 1988. One year before, the artwork had been shut down due to failure and fire hazard of the monitors. The result of my study was that a sustainable solution could only be the complete replacement of the wiring and the obsolete CRT monitors, which had already been changed several times before with new models that were both aesthetically and historically unsuitable, with cylindrical, continuous LED panels of the latest generation. This serious intervention due to the absolute lack of 1,003 authentic CRT monitors on today's equipment market, also due to the perpetual repair of these defective monitors to be anticipated, was also motivated by the approach to such cases communicated to me years before by the artist himself: "Go with the flow". In accordance with the artist's thinking, I called the solution I proposed "reincarnation" because it reproduces the artist's idea, indeed the spirit of this installation, in a new body of equipment. Years before, as head of the collection of the ZKM | Medienmuseum, I had only allowed the strict exchange of absolutely identical devices. At that time, this was only possible because we only needed individual monitors for individual works in the respective original casings, which we had previously stored in larger quantities. But here, with a demand for 1,003 monitors of various models ans sizes, produced in 1988 or shortly before, this was definitely no longer possible. In view of the ethical recognition of the high value of cultural memory, this project called "reincarnation" could be evaluated as a model project for the preservation of numerous related artworks using obsolete equipment. 
As I have learned recently, the National Museum has decided against Reincarnation and chosen to repair the old monitors, which are already no longer original, or if necessary, to replace them with similar monitors. As could be expected, this work encounters almost insurmountable technical problems. The future will show whether the repair of the CRT monitors has led to a reliable and sustainable solution.

\section{The value of aesthetic integrity}

On the question of aesthetic integrity we come to no different conclusion, for the latter is intrinsically linked to physical integrity. According to our traditional ethical codes, and even more so with regard to the artist's intention, personal style and ever-praised signature, we are first of all urged to establish the strictest rules in this regard. An example may explain this more simply. The aesthetics of an analogue black-and-white video, accompanied by noise and multifarious artifacts, recorded on a quarter-inch Akai VT-110 device, such as Joseph Beuys' Boxing Match for Direct Democracy by Referendum, at documenta 5 in 1972, with the duration of 9:30 minutes, played back on a small black-and-white monitor from the same period, can never be matched by the copy of that very video, which was later converted several times and then digitized, and is now played back on a contemporary LED monitor. This is so obvious that it does not require proof.

However, the serious as well as troublesome question arises, if at all we have such a valuable video in our collection, what compromises we have to make, also and especially with regard to the aesthetic integrity of the artwork, in order to make this video visible at all today. The in-depth discussion of conservation-restoration issues is the subject of the following contributions to this symposium. With this example, I would like to illustrate in advance only on what slippery ground we immediately move when we apply to the conservation or even digitization of video artworks the standards that traditionally apply to painting, sculpture, and drawing.

It should also be clearly stated here that the digital transformation of an analogue work is never the work itself, but only a substitute and nothing more, in a different and reduced format than the original, which can only be made visible by means of digital 
technology, which does not have the same aesthetic qualities as analogue technology. It is, at best, a kind of a viewing copy of the art work.

However, this insight cannot absolve us from the task of saving such a time-based work from disappearing. Obviously, however, this task brings us into an ethical dilemma that we can solve only if we abandon some of our traditional ideals and aspirations in order to preserve at least the core of a work. And it is precisely in this sense that we must strive for a new conception of the ethical values of our work and be aware that in the future we will often show no more than copies or even simulations, but not the works of art themselves.

At this point it should be further clarified that, in my opinion, we are still in no way allowed to "improve" a work of art by virtuoso technical manipulations, to "beautify" it, or to make it more "efficient" in its functions. This is also the case when the artist expressly requests it. For we remain, if I am not mistaken, the guardians of the work in its most authentic form, even if we are often confronted with difficulties during exhibition presentations that could be solved by some technical improvements. Admittedly, this can only apply as long as the material and technical conditions corresponding to the authentic form have not yet been definitively lost.

With this likewise ethical question of fidelity to the work, we are moving into the domain of a controversy that has been almost painful for decades and for which we have not yet found a solution. The well-intentioned motto "as little intervention as necessary" does not help if entire technologies have disappeared and if we are obviously not able to replace them adequately without affecting the character of the work.

Example: At Gulbenkian Foundation in Lisbon I found one of the wonderful "Paseos" of Francis Alys, of his early wanderings through Mexico City, filmed in $16 \mathrm{~mm}$. The image quality attracted me and the supposed humming of the $16 \mathrm{~mm}$ projector behind me, right above my head, put me in the mood of my own experiments with $16 \mathrm{~mm}$ cameras. I can't remember the exact title of the work, but I know for sure that the work was labeled $16 \mathrm{~mm}$ film in its original medium. And when something irritated me about 
the brilliant, harshly contrasted image, I went to the projection screen and was disappointed to discover that it was a simple video projection. I discovered the source of the sound on the side of the video projector, a cheap little loudspeaker on which the sound of a $16 \mathrm{~mm}$ film projector was played. What a disappointment. I felt cheated by this shabby simulation of Alys' work and left the exhibition disappointed. Quite clearly, it is still possible to show $16 \mathrm{~mm}$ films with an appropriate film projector. The rental and maintenance of the projector as well as the constantly necessary commissioning of film copies is indeed significantly more expensive than playing digital data on a video projector. But can and may budget constraints serve as justification for a falsifying presentation of a work, in which the viewer is also deliberately made to believe, through the simulated sound, that the work is presented with a film projector?

Budget constraints naturally lead to the next dilemma facing curators and conservators. The choice is very much one of showing a work of art in the best possible authentic form at great cost - or of foregoing the presentation of that work of art altogether because of that cost. And at this point, too, questions of conservation ethics must be discussed again.

\section{The value of historical integrity}

With the partial or complete replacement of obsolete equipment with more modern ones, which often enough change the appearance, functionality and aesthetics of the work, we also risk sacrificing the value of historical integrity that has been so well guarded until now. No sane conservator would paint over the defects of an 18thcentury oil painting with modern acrylic paint, even if this were technically the more durable solution and it would not even be seen. Historical integrity was and is not meant to simulate an earlier state, but to preserve the work in its exact physical and aesthetic integrity.

Example: When an analog $16 \mathrm{~mm}$ film from the early $1990 \mathrm{~s}$ is transferred to digital data, which is then played back by a digital projector, the historical properties inscribed in the original are replaced by those that the work could not have had at the time of its creation. For this reason, the historical origins of a $16 \mathrm{~mm}$ film are no longer 
recognizable to today's viewers if it has been digitally simulated. Such a video could just as well be a recording from today.

Example: If the original CRT monitors in Nam June Paik's 1988 work The More the better were still present at all, and if they were re placed today by a newer generation of CRT monitors, now recorded with the digitized data of the original analogue videos, neither the technology nor the aesthetics of the late 1980s would be recognizable to the visitor. However, such a manipulated work would willfully deceive the visitor and make him believe that it is the genuine historical work. The only remedy could be to inform the visitor precisely with the work label that it is a technical reconstruction of the work. So far, most museums and collections have shied away from disclosing such reconstructions.

It is indicative of the uncertainties associated with the current shift in values that we often miss, both in presentations and in publications, a sharp distinction between the terms we use to describe our work. If preservation is still the most neutral term, conservation, restoration, restitution, documentation, migration, retrieval, saveguarding, iteration, recreation, reenactment, reinstallation ... etc. are all terms that have been used to describe our work. Meaningful terms that have always been interpreted differently by different professions throughout history. However, this vagueness of the term increases when preservation practices from decades or even centuries, are no longer possible within a decade for trivial technical reasons. And here again, it seems, digitization is seen as a remedy that can solve every problem, even in our field. What is fatally overlooked is that digitization, in its extreme dependence on industrially manufactured hardware and software, is often enough itself the cause of the urgently needed adjustments.

Under these conditions, it seems as if we would have to sacrifice again and again not only the preservation of the physical and aesthetic integrity, but also the historical integrity of the work. If, in this dilemma, we remember that the substance of art lies in the idea, which can undoubtedly be applied to the individual work of art, then it seems to me that the application of the concept of "reincarnation" to those works for the preservation of which we once or constantly depend on the exchange of software and 
hardware makes sense. In reference to the traditional restoration ethics, it would then be necessary and crucial that we finally say goodbye to the terms "originality" and "authenticity" and tell the viewer, visitor, user in all openness that it is a reincarnation of the original work, in which the original idea appears in a new body. It is obvious that this would entail further consequences that will have to be discussed in the near future.

\section{Conclusion: Further questions and encouragement}

Given the factual technical difficulties and only slowly growing knowledge in the field of preservation and restoration of electronic and digital media art, most museums and collections today are hardly in a position to uphold the noble standards that traditional preservation ethics demanded. The question therefore also arises whether they are still obliged to do so at all in a situation of growing demands and dwindling resources. Can the works themselves and the supposed enduring value of contemporary art collections even be preserved over longer periods of time? And if the limits of conservation practice, especially in the case of digital art, mean that only a few works will survive, what criteria and preferences should be used to decide which works should be passed on while the rest are allowed to perish? It is to be feared that the technical development and thus the deterioration of these works will proceed so quickly that we will not even have the time to make such an assessment.

The picture I paint here is somber, as I look not only at the well-funded flagship museums and collections, but also at the many ordinary museums that are moderately funded at best, but where much of contemporary art is preserved. We must also preserve these many works of art and make them accessible by the best possible means. The way to do this is to renegotiate ethical standards that have been maintained up to now. In doing so, however, it would be unacceptable if we had to abandon these because our institutions are no longer in a position or willing to provide the resources to do so. Therefore, in addition to our daily conservation work, it is equally important that we make every effort to improve our overall institutional funding and working conditions so that our daily work can be sustainably fruitful. 